\title{
El mapa de las desigualdades en salud por grupos de edad en España*
}

\author{
Alexandrina Stoyanova \\ Departament de Economia i Empresa, Universitat Pompeu Fabra \\ y Centre de Recerca en Economia del Benestar, Universitat de Barcelona \\ Marisol Rodríguez \\ Departament de Política Econòmica i Estructura Econòmica Mundial \\ y Centre de Recerca en Economia del Benestar, Universitat de Barcelona \\ Jaime Pinilla \\ Departamento de Métodos Cuantitativos en Economía y Gestión, \\ Universidad de las Palmas de Gran Canarias
}

\section{Resumen:}

La existencia de desigualdades socioeconómicas en salud está muy documentada, tanto en nuestro país como en otros países, sobre todo europeos. Los análisis han cubierto también las diferencias regionales y de género. Sin embargo, no hay ningún trabajo en España que haya estudiado las desigualdades socioeconómicas en salud por grupos de edad de forma sistemática. Este artículo viene a cubrir esa laguna, utilizando la Encuesta Nacional de Salud de 2003, a cuyos datos se aplica la metodología de los indices de concentración. Los resultados arrojan un perfil de U invertida. Esto es, las desigualdades son menores en el caso de los adultos jóvenes y los de más de 74 años, y son mayores en las edades previas a la jubilación. Las dos variables que más contribuyen a dicha desigualdad, para el grupo de edad entre 55 y 64 años, son la propia renta y la educación superior. Estos resultados pueden guiar las politicas paliativas de las desigualdades hacia dianas mejor identificadas. De todas formas, en la discusión se llama la atención sobre el carácter puntual y no longitudinal de los datos, que impide hacer juicios sobre la posible evolución de las desigualdades en el tiempo.

Palabras clave: desigualdades en salud, grupos de edad, España

Clasificación JEL: D63, I10, I12

\begin{abstract}
:
Income related inequalities in health and inequities in health care utilization are well documented, both in Spain and other countries, above all in Europe. The studies have also dealt with regional and gender differences. However, the study of those inequalities by age groups in a systematic fashion, has not been done yet in Spain. This research tries to cover this gap, using data from the National Health Interview Survey of 2003, to which we apply the methodology of concentration indices, widely used in health economics. The results show a profile of inverted $U$. The largest concentration indices are found for ages previous to retirement, while inequalities are smaller among young and oldest adults. The two variables with the highest contribution to inequality in self assessed health in the age group 55-64 years old, are income and higher education. These results can guide policy to better identified targets. Anyways, at-
\end{abstract}

* Los autores agradecen la ayuda del FIS, proyecto 03/1742, así como las sugerencias y comentarios de Ángel López Nicolás. 
tention is drawn to the fact that we work with cross-sectional and not longitudinal data, which makes it difficult to pre-judge how inequalities will evolve in the future.

Key words: health inequalities, age groups, Spain

JEL Classification: D63, I10, I12

\section{Introducción}

A pesar de que la mayoría de los países europeos disfrutan de sistemas públicos de atención sanitaria, con cobertura universal en prácticamente todos ellos, desde hace ya bastantes años se viene documentando la existencia de desigualdades pronunciadas en el estado de salud entre diferentes grupos socioeconómicos, siendo el Informe Black (Townsend, Davidson, Whitehead, 1992), publicado en 1980, el estudio con el que se inició esta línea de investigación y análisis. En general, los individuos que tienen una posición educativa más elevada, pertenecen a estratos sociales más altos o disponen de mayor nivel de ingresos tienen una mejor autovaloración de la salud, presentan menor grado de morbilidad e inferiores tasas de mortalidad. Como vemos, las desigualdades se manifiestan usando cualquiera de los indicadores de nivel socioeconómico y cualquiera de los indicadores disponibles de salud. Por otro lado, este es un terreno de contribución fructífera tanto de economistas de la salud como de médicos epidemiólogos y otros profesionales sociales. Entre los economistas, los trabajos más conocidos en Europa son los del grupo ECuity (véase, por ejemplo, Van Doorslaer, Wagstaff et al., 1997; Van Doorslaer y Koolman, 2004). Entre los epidemiólogos y otros analistas sociales destacamos los trabajos de Kunst y Mackenbach (Kunst et al., 1998; Mackenbach y Kunst, 1997), Marmot (Marmot et al., 1991) y Wilkinson (Wilkinson, 1996; Wilkinson 1997), aunque la lista se podría ampliar con muchos otros trabajos de estos mismos autores y otros más.

En España, los numerosos estudios realizados a lo largo de las dos últimas décadas no sólo han puesto en evidencia la existencia de desigualdades sociales y de género en salud (Regidor et al., 1994; Benach et al., 1996; Navarro y Benach, 1996; Borell y Benach, 2003; Borrell et al., 2004), sino que también han analizado la evolución de dichas desigualdades a lo largo del tiempo y han desvelado la presencia de considerables diferencias regionales (Urbanos, 2000; García y López-Nicolás 2007a y 2007b; Cantarero y Pascual, 2005).

Sin embargo, el estudio de las desigualdades en salud por grupos de edad ha recibido menos atención, sobre todo dentro de nuestro país. Una idea muy extendida es que las desigualdades socioeconómicas en salud comienzan ya en el útero de la madre (debido a factores biológicos y sociales) y se mantienen a lo largo de la vida. Algunos autores (West, 1997) han mostrado, no obstante, que durante la juventud se produce una igualación de la salud entre todas las clases sociales. Asimismo, otros autores (Victor, 1989) han argumentado que la vejez es una época que se caracteriza por la universalización de la mala (menos buena) salud y han excluido expresamente a la población 
envejecida de sus análisis o han predicho una reducción de las desigualdades a medida que la probabilidad acumulada de muerte para cada individuo se acerca al 100\% y entonces los determinantes sociales u otros factores de riesgo discriminarían peor. En esta línea, hay algunos estudios recientes, que analizan el nivel de salud de las personas mayores, a menudo haciendo énfasis en el impacto que la jubilación puede tener en dicha salud. Por ejemplo, Marmot y Shipley (1996) utilizan datos del Whitehall study $I^{1}$ para analizar si las diferencias socioeconómicas (en este caso, por tipo de ocupación) en mortalidad se dan también después de la jubilación. Su conclusión es que las diferencias absolutas en mortalidad (sin controlar por otras variables) entre el nivel de ocupación más alto y el más bajo aumentan con la edad, pero las diferencias relativas disminuyen después de la jubilación (lo que sugiere que trabajar o no contribuye a dichas desigualdades). Huisman, Kunst y Mackenbach (2003), en un análisis sobre once países europeos, encuentran que las desigualdades en morbilidad disminuyen con la edad a partir de los 60 años, pero no desaparecen del todo. Una reducción significativa de la desigualdad en el estado de salud autopercibido es, asimismo, lo que halla Van Ourti (2003) para los belgas mayores de 65 años. Sin embargo, Chandola et al. (2007), con datos del Whitehall Study II, hallan que las desigualdades se ensanchan con la edad, debido a que los individuos en las ocupaciones más bajas tienen una tasa de deterioro de la salud física mayor que la tasa de deterioro de los individuos en las ocupaciones más altas.

El principal objetivo de este trabajo es trazar el mapa sistemático de las desigualdades en salud por grupos de edad en España. Esta es una tarea que estaba pendiente y que puede ser útil, sobre todo en la toma de medidas para reducir desigualdades, al permitir dirigir los esfuerzos hacia dianas mejor identificadas. Pretendemos, por tanto, cuantificar las desigualdades en salud para diferentes grupos de edad y comparar sus magnitudes para observar si las desigualdades son mayores o menores en unos grupos que en otros. Advertimos, en seguida, que se trata de una fotografía instantánea y no de una evolución en el tiempo. En este sentido, nuestro objetivo es más abrir una línea de investigación que dar una respuesta definitiva a las muchas preguntas que se pueden hacer relativas a este tema. Mediremos la salud según tres indicadores: salud percibida, presencia de al menos una enfermedad crónica y limitaciones en las actividades habituales debido a problemas crónicos o enfermedades de larga duración. Las desigualdades se miden mediante el índice de concentración. Además de cuantificar las desigualda-

El Whitehall study I es un estudio longitudinal que consiste en el seguimiento durante aproximadamente 25 años de 18.133 hombres, trabajadores del sector público, destinados en unidades gubernamentales situadas en Londres, a los que se les hizo una serie de pruebas iniciales de salud en 196770. El Whitehall study II se inició en 1985 y está previsto que finalice en 2009. Se deriva del anterior estudio introduciendo algunas modificaciones, siendo la principal de ellas la inclusión de mujeres en el seguimiento. Aunque el objetivo inicial no era el análisis de las desigualdades en salud, éste es en realidad el tema por el que más se conoce este estudio, pues ha puesto claramente de relieve que las diferencias de mortalidad (salud) no son simplemente consecuencia de la pobreza, sino que existe un gradiente social claro en la mortalidad y otros indicadores de salud. Además del impacto de la ocupación, los trabajos asociados a este estudio están intentando dilucidar el papel de otras variables que ayuden a explicar dicho gradiente social. 
des, un segundo objetivo, de igual o mayor interés para la política sanitaria, es descomponer las desigualdades halladas en sus factores explicativos.

Teniendo en cuenta la literatura previa, nuestra hipótesis de partida es que en las edades adultas jóvenes hay relativamente pocas diferencias en el estado de salud y que éstas se tornan substanciales en el auge de la madurez, para volver a descender en la vejez, a partir de los 65 años. Las desigualdades tendrían, por consiguiente, una forma de U invertida. Deaton y Paxson (1998) también encuentran, para Estados Unidos, que la dispersión (la varianza) en la distribución conjunta de la renta y el estado de salud autopercibido aumenta con la edad, hasta llegar a la edad de jubilación, y a partir de ahí se reduce. Es más, la correlación entre renta y salud varía con la edad: es pequeña para los adultos jóvenes (en los primeros años de la veintena) y después va creciendo de forma sostenida hasta alcanzar un máximo alrededor de los 50-60 años.

\section{Metodología}

\subsection{El cálculo de las desigualdades socioeconómicas en salud}

La metodología, ahora estándar, en el estudio de las desigualdades socioeconómicas en salud es medir éstas mediante el índice de concentración (Wagstaff, Paci y Van Doorslaer, 1991). Para una variable o indicador de salud $y_{i}$ continuo la curva de concentración $L(s)$ (véase la figura 1) muestra la distribución acumulada de salud frente la proporción acumulada de la población, ordenada ésta por el nivel de renta (de menor a mayor). Si $L(s)$ coincide con la diagonal la salud está distribuida de forma equitativa entre la población, es decir, cada individuo disfruta del mismo nivel de salud (sea éste bueno o malo). Si la curva de concentración se encuentra por debajo de la diagonal eso será indicativo de que la variable $y_{i}$ está menos concentrada en los primeros grupos de población (los de menor renta) y más concentrada en los últimos grupos (los de mayor renta). Esto es lo usual cuando $y_{i}$ mide, por ejemplo, la buena salud. Sin embargo, cuando $y_{i}$ mide algún indicador «negativo», de mala salud, lo habitual es que esté proporcionalmente más concentrada en los grupos de menor renta, y en ese caso la curva de concentración $L(s)$ discurrirá por encima de la diagonal. Cuanto mayor es el área entre $L(s)$ y la diagonal, tanto mayor es el grado de desigualdad. Finalmente, hay que tener en cuenta que, por convención, al área por debajo de la diagonal se le asigna signo positivo, y al área por encima de la diagonal signo negativo. Por lo tanto, un signo positivo indica que existe desigualdad a favor de los más ricos; es decir, estos individuos acumulan una proporción relativamente más alta de la variable en cuestión. Al contrario, un signo negativo indica que son los más pobres los que tienen una proporción relativamente más alta del indicador de salud representado.

A partir de aquí, el índice de concentración se define como dos veces el área entre la curva de concentración y la diagonal y toma valores entre - 1 y 1 . Los extremos indican máxima desigualdad en la distribución de la salud, es decir, toda la buena o mala 
FIGURA 1

\section{CURVA DE CONCENTRACIÓN}

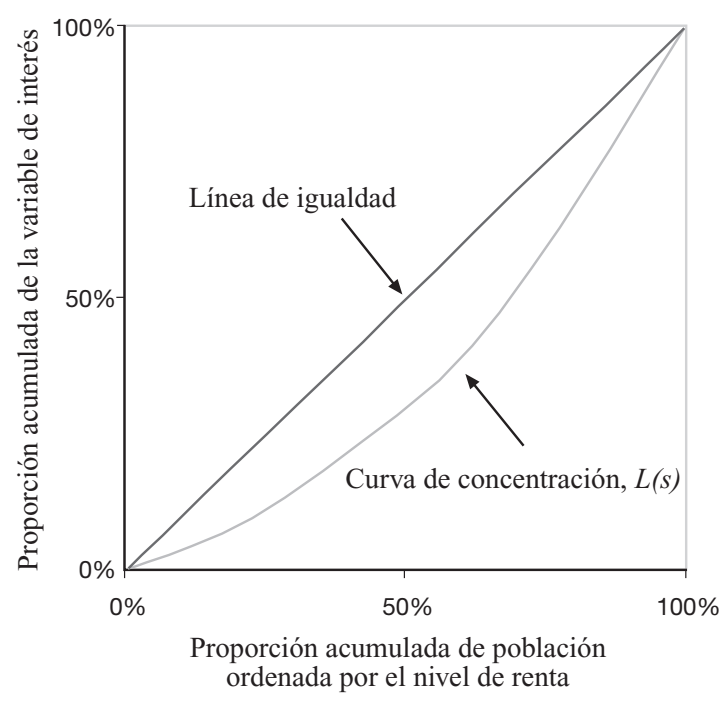

salud de la población está concentrada en manos del más rico o del más pobre de la población. Un valor cero o cercano a cero indica ausencia de desigualdad o que ésta es muy pequeña. Formalmente el índice de concentración se puede expresar como:

$$
I C=1-2 \int_{0}^{1} L(s) d s
$$

El cálculo del índice de concentración puede realizarse utilizando la fórmula de la covarianza (Kakwani, Wagstaff y Van Doorslaer, 1997):

$$
I C=\frac{2}{N \mu} \sum_{i=1}^{N} y_{i} R_{i}-1=\frac{2}{\mu} \operatorname{cov}\left(y_{i}, R_{i}\right),
$$

donde $\mu=\frac{1}{N} \sum_{i=1}^{N} y_{i}$ es la media de la variable de salud, $N$ es el tamaño de la muestra y $R_{i}$ es la posición relativa del individuo $i$ en la distribución de la renta.

Una forma alternativa de obtener el índice es la denominada regresión conveniente, consistente en aplicar mínimos cuadrados ordinarios (MCO) a la siguiente ecuación:

$$
2 \sigma_{R}^{2}\left[\frac{y_{i}}{\mu}\right]=\alpha+\beta R_{i}+\varepsilon_{i},
$$

donde $\sigma_{R}^{2}$ es la varianza de la variable $R_{i}$ y $\varepsilon_{i}$ es el término de error. El coeficiente $\beta$ estima directamente el índice de concentración. 


\subsection{Descomposición de las desigualdades}

Una vez cuantificado el grado de desigualdad en salud, tiene especial interés conocer los factores que causan las desigualdades y determinar cual es la contribución de cada uno de ellos. Siguiendo el método propuesto por Wagstaff, Van Doorslaer y Watanabe (2003), especificamos un modelo de regresión lineal que explique el indicador de salud, $y_{i}$, en función de $k$ determinantes exógenos.

$$
y_{i}=\alpha+\sum_{i=1}^{N} \beta_{k} x_{k i}+\varepsilon_{i} .
$$

Aplicando el teorema de Rao para la desigualdad de la renta, el índice de concentración se puede descomponer en factores, tal como aparece a continuación:

$$
I C=\sum_{i=1}^{N}\left(\beta_{k} \bar{x}_{k i} / \mu\right) C_{k}+\frac{G C_{\varepsilon}}{\mu},
$$

donde $\bar{x}_{k}$ es la media de la variable $x_{k}, C_{k}$ es su correspondiente índice de concentración (que se define de forma similar al $I C$ ), y $G C_{\varepsilon}$ es el componente residual que refleja la desigualdad en salud que no se puede explicar por la variación sistemática de los regresores entre los diferentes grupos de renta. Sin embargo, cuando se utiliza la regresión por intervalos para convertir la variable dependiente en continúa, los residuos no se pueden calcular y por tanto este último término desaparece de la ecuación. La anterior formulación indica que el índice de concentración, $I C$, es una suma ponderada de los índices de concentración de los $k$ regresores (calculados con respecto a la renta como variable de ordenación), donde los pesos son las elasticidades de la variable endógena $y$ con respecto a cada $x_{k}$ (evaluada en la media). La elasticidad se puede escribir como $\hat{\eta}_{k}=\hat{\beta}_{k} \bar{x}_{k} / \mu$ y mide el impacto que cada determinante $k$ tiene sobre la salud. Por tanto, la descomposición se puede re-escribir como:

$$
I C=\sum_{k} \hat{\eta}_{k} \hat{C}
$$

\section{Descripción y tratamiento de los datos}

Los datos empleados en este trabajo proceden de la Encuesta Nacional de Salud (ENS) de 2003. La ENS ser refiere a una muestra representativa de toda la población española y contiene información sobre el estado de salud general y los principales problemas asociados con éste (enfermedades crónicas, dolencias y limitaciones de la actividad cotidiana, accidentes), así como sobre la utilización de los servicios sanitarios, el uso de determinadas prácticas preventivas y los hábitos que suponen riesgo para la salud de los entrevistados. Asimismo, proporciona información sobre varios aspectos 
demográficos y socioeconómicos tanto de la persona entrevistada como del resto de los miembros del hogar. Hemos utilizado la muestra de adultos (mayores de 15 años) que contiene 21.650 observaciones. Con el propósito de detectar desigualdades en salud por edad hemos definido siete grupos de edad: 16-24 años, 25-34 años, 35-44 años, 45-54 años, 55-64 años, 65-74 años y 75 años y más. Todos los análisis empíricos se han llevado a cabo para cada uno de estos grupos.

Como medidas de la salud hemos considerando tres indicadores: el estado de salud percibido, la presencia de al menos una enfermedad crónica de entre las definidas en la encuesta, y la existencia de limitaciones en las actividades habituales como consecuencia de esa o esas enfermedades crónicas. La variable que recoge la autovaloración de la salud es uno de los indicadores más utilizados en el estudio de las desigualdades socioeconómicas en salud. A pesar de ser una medida subjetiva y sencilla, se ha demostrado su poder como predictor de la esperanza de vida, de la mortalidad y de la utilización de servicios sanitarios (Idler y Beyamini, 1997; Burström y Fredlund, 2001; Van Doorslaer y Gerdthan, 2003). Sin embargo, el carácter ordinal que toma la respuesta a esta pregunta en la ENS impone dificultades teóricas y cuantitativas, derivadas de su no continuidad, que conviene analizar y corregir.

En la ENS las respuestas posibles van desde 1 (muy buena) hasta 5 (muy mala). Para el empleo de esta variable en el cálculo de los índices de concentración y descomposición de los mismos, algunos autores proponen dicotomizar la variable entre buena y no buena salud. Sin embargo, esta dicotomización, además de no conseguir la continuidad, añade la desventaja que supone no utilizar todos los posibles estados de salud, lo que provoca que las comparaciones de desigualdad en el tiempo o entre poblaciones sean poco fiables (Van Doorslaer y Koolman, 2004).

Otra estrategia consiste en estimar una variable latente continua e inobservable, subyacente en la distribución empírica de las respuestas a la autovaloración de salud. Para ello se utilizan modelos probit o logit ordenados que tienen como variable dependiente la autovaloración de la salud del individuo. Las predicciones lineales de la variable latente se pueden utilizar como medidas del estado de salud de los individuos.

Una alternativa de estimación más eficiente que los modelos ordenados la proporciona la regresión por intervalos, cuando los valores de los extremos de los intervalos son conocidos. Cuando no se conocen, se pueden asignar utilizando, por ejemplo, la metodología de Van Doorslaer y Jones (2003) basada en la correspondencia entre la distribución del índice de utilidad de la salud (Health Utility Index, $\mathrm{HUI}^{2}$ ) y las categorías de la variable salud autopercibida en la encuesta de salud canadiense. A partir de los valores del HUI y mediante regresión por intervalos, los autores imponen

2 El HUI es una medida de salud que se obtiene convirtiendo la información sobre siete dimensiones relacionadas con la salud — sentidos (visión, oído, habla), movilidad, cognición, autocuidado, estado emocional, dolor o malestar y fertilidad - en unidades de utilidad (utility scores) a través de una función de utilidad basada en una escala genérica donde muerto es igual a 0 y estar en perfecto estado de salud es igual a 1 . 
cardinalidad a la variable de salud ordinal tomando como extremos para cada categoría los puntos de corte de la función de distribución empírica del HUI. Puesto que, al contrario que la encuesta de salud canadiense, la mayoría de las encuestas de salud europeas no disponen de ambas medidas de salud, muchos autores han seguido el método de Van Doorslaer y Jones (2003) para convertir la variable salud autopercibida en una variable continua, partiendo del supuesto de que la distribución de la salud entre la población canadiense es una buena aproximación de la distribución de la salud en Europa.

En nuestro caso, tampoco disponemos de información que nos permita calcular el índice de utilidad. Además, consideramos que utilizar los valores de la encuesta canadiense de 1994 es una aproximación bastante imperfecta. Por ello, para cardinalizar la variable de salud ordinal, hemos optado por realizar la represión por intervalos utilizando como extremos de los intervalos los propios límites de la frecuencia acumulada en cada una de las cinco categorías del estado de salud autopercibido. La posterior predicción nos facilita un índice de salud continuo para cada individuo.

La segunda medida de salud que emplearemos en el análisis es la presencia de enfermedades crónicas. Éste es un indicador objetivo de la salud y recoge trastornos de larga duración. La ENS contiene información sobre varias condiciones crónicas (hipertensión arterial, colesterol elevado, diabetes, asma, enfermedad del corazón, úlcera de estómago, alergia, artrosis, problemas circulatorios, depresión, y migrañas). Basándonos en estos datos, hemos definido una variable dicotómica que toma el valor 1 si el individuo declara tener al menos uno de estos trastornos crónicos. Para ofrecer una medida más exacta de la cronicidad (y posiblemente más indicada para el análisis de las desigualdades) sería deseable disponer de un índice ponderado por la gravedad de los diferentes problemas crónicos. Sin embargo, la ENS no facilita la información necesaria para obtenerlo. Los resultados descriptivos indican que más de la mitad de los adultos sufre al menos una enfermedad crónica. Las condiciones crónicas afectan a casi uno de cada cuatro jóvenes entre 16 y 24 años, y cerca de 9 de cada 10 mayores de 74 años. Es decir, la prevalencia de enfermedades crónicas exhibe un patrón creciente con la edad. Sin embargo, la relación entre cronicidad y la edad no está tan clara si se consideran separadamente las distintas enfermedades crónicas. Un examen de los datos (que no se muestra en las tablas) revela que, comparado con el resto de grupos de edad, los mayores de 74 años presentan el mayor riesgo de padecer hipertensión ( $43,2 \%$ de los individuos del grupo), diabetes (16,9\%), enfermedades del corazón (23,5\%), asma (13,7\%), artrosis $(48,7 \%)$ y mala circulación $(31,8 \%)$. Todas ellas crecen con la edad. La prevalencia del colesterol elevado, los problemas relacionados con dolores de cabeza y la depresión aumenta hasta los 65 años y desciende para el grupo de los mayores de 74 años. Por el contrario, las alergias están mucho más presentes entre los jóvenes entre 16 y 24 años (14,4\%).

La información sobre la cronicidad se completa con una nueva variable dicotómica, que recoge si el individuo declara o no padecer algún tipo de limitación debido a la enfermedad crónica. Este constituye nuestro tercer indicador de salud. Los datos muestran que la reducción de las actividades habituales debido a trastornos cró- 
nicos es elevada $(19,2 \%)$, mayor entre las mujeres que entre los hombres $(23,1 \%$ y $14,7 \%$, respectivamente) y creciente con la edad ( $7,1 \%$ entre los más jóvenes frente a 42,8\% entre los mayores de 74 años).

Para el cálculo de los índices de concentración se ha utilizado como variable de ordenación la renta equivalente del hogar. Esta misma variable se utiliza también como explicativa en la descomposición de las desigualdades. Dado que los ingresos mensuales del hogar se recogen en la ENS mediante una variable ordinal de ocho categorías y la tasa de no respuesta es alta (alrededor del 20\%), hemos imputado la renta familiar utilizando el método de regresión por intervalos. En la regresión controlamos por diferencias en características individuales como la edad, sexo, estado civil, educación, situación laboral, ocupación, Comunidad Autónoma y tamaño del municipio de residencia. Para convertir los ingresos del hogar, una vez imputados, en renta equivalente por individuo hemos aplicado la escala de equivalencia de la OCDE modificada ${ }^{3}$. La media de la renta equivalente del hogar asciende a 705 euros mensuales, y su distribución por grupos de edad muestra valores medios más elevados en las edades 25-34 y 35-44 años (véase la Tabla 1).

El análisis incluye también otras variables explicativas de las desigualdades en salud. Todas ellas, así como sus valores medios para cada franja de edad y el total de la muestra, aparecen también en la Tabla 1, que comentamos a continuación.

Por lo que respecta a la educación, a partir de las 10 escalas iniciales que incluye la ENS se han definido cuatro variables dicotómicas que reflejan el nivel más alto de estudios terminados por el entrevistado: sin estudios (14,8\% de la muestra), educación primaria $(35,6 \%)$, educación secundaria $(31,2 \%)$ y educación universitaria $(18,4 \%)$. Las diferencias en el perfil educativo de los distintos grupos de edad son marcadísimas, como era de esperar en un país que ha evolucionado muchísimo en los últimos cincuenta años. Por su parte, la situación laboral se describe a través de ocho variables dicotómicas. Este gran nivel de detalle permite distinguir entre los distintos tipos de pensiones (de jubilación, de invalidez u otros) los cuales, probablemente y a tenor de lo visto en la literatura, tendrán un impacto diferencial sobre las desigualdades socioeconómicas en salud. Un 15\% de los individuos incluidos en la muestra son jubilados, 3,3\% perciben pensiones de invalidez y el 7,7\% son perceptores de otro tipo de pensiones (viudedad, orfandad, etc.). Lógicamente, estas variables relativas a la situación laboral cambian mucho según la franja de edad.

En cuanto al estado civil, existe amplia evidencia empírica que demuestra los beneficios que tiene sobre la salud el hecho de estar casado o no (Gardner y Oswald, 2004; Hu y Goldman, 1990; Wyke y Ford, 1992). Los hombres y las mujeres casados declaran mejor estado de salud percibida, presentan menores tasas de enfermedades de larga duración y son más longevos que los individuos en otros estados civiles. Cabe, pues, esperar que el estado civil sea una de las posibles explicaciones de las desigual-

Según la escala de equivalencia de la OCDE modificada los pesos se distribuyen de la siguiente forma: 1 para el primer adulto, 0,5 para el resto de personas mayores de 14 años y 0,3 para cada niño de menos de 14 años. 
TABLA 1

DESCRIPCIÓN DE LAS VARIABLES POR GRUPOS DE EDAD

\begin{tabular}{|c|c|c|c|c|c|c|c|c|}
\hline & $\begin{array}{c}16-24 \\
\text { años }\end{array}$ & $\begin{array}{c}25-34 \\
\text { años }\end{array}$ & $\begin{array}{c}35-44 \\
\text { años }\end{array}$ & $\begin{array}{l}45-54 \\
\text { años }\end{array}$ & $\begin{array}{l}55-64 \\
\text { años }\end{array}$ & $\begin{array}{l}65-74 \\
\text { años }\end{array}$ & $\begin{array}{c}75+ \\
\text { años }\end{array}$ & Total \\
\hline Número de observaciones & 2.092 & 3.240 & 4.324 & 3.102 & 2.758 & 3.321 & 2.813 & 21.650 \\
\hline Salud (\%) & & & & & & & & \\
\hline Muy buena salud & 17,26 & 14,85 & 12,05 & 9,16 & 6,02 & 3,67 & 2,88 & 9,31 \\
\hline Buena salud & 69,31 & 69,29 & 67,21 & 60,06 & 47,97 & 39,57 & 33,27 & 55,60 \\
\hline Salud regular & 11,71 & 13,27 & 16,35 & 22,79 & 32,89 & 40,35 & 41,06 & 25,36 \\
\hline Mala salud & 1,34 & 2,16 & 3,42 & 6,54 & 9,72 & 13,16 & 17,53 & 7,61 \\
\hline Muy mala salud & 0,38 & 0,43 & 0,97 & 1,45 & 3,41 & 3,25 & 2,36 & 2,12 \\
\hline Enfermedad crónica (\%) & 23,47 & 27,41 & 33,72 & 47,97 & 70,70 & 85,53 & 87,98 & 53,23 \\
\hline $\begin{array}{l}\text { Limitación por enfermedad } \\
\text { crónica }(\%)\end{array}$ & 6,21 & 7,13 & 9,76 & 15,99 & 26,32 & 32,19 & 38,93 & 19,26 \\
\hline $\begin{array}{l}\text { Renta del hogar equivalente } \\
\text { (euros) }\end{array}$ & $605 €$ & $793 €$ & $793 €$ & $744 €$ & $700 €$ & $633 €$ & $592 €$ & $705 €$ \\
\hline Educación (\%) & & & & & & & & \\
\hline Sin estudios & 2,01 & 3,09 & 3,42 & 6,74 & 17,11 & 32,01 & 41,70 & 14,81 \\
\hline Primaria & 16,20 & 17,01 & 25,07 & 38,65 & 52,94 & 52,88 & 47,03 & 35,62 \\
\hline Secundaria & 66,59 & 44,26 & 42,90 & 33,88 & 18,49 & 9,73 & 6,58 & 31,18 \\
\hline Universitaria & 15,20 & 35,65 & 28,61 & 20,73 & 11,46 & 5,39 & 4,69 & 18,38 \\
\hline Situación laboral (\%) & & & & & & & & \\
\hline Trabaja & 32,41 & 69,66 & 72,73 & 64,38 & 36,84 & 1,78 & 0,11 & 42,28 \\
\hline Parado & 10,09 & 9,26 & 5,32 & 4,74 & 4,28 & 0,06 & 0,00 & 4,66 \\
\hline Jubilado & 0,00 & 0,00 & 0,07 & 0,93 & 10,51 & 46,82 & 46,14 & 14,67 \\
\hline $\begin{array}{l}\text { Percibe pensión por } \\
\text { invalidez }\end{array}$ & 0,38 & 1,20 & 1,90 & 4,16 & 8,09 & 4,85 & 3,20 & 3,38 \\
\hline $\begin{array}{l}\text { Percibe otras pensiones } \\
\text { (viudedad, orfandad, etc.) }\end{array}$ & 0,19 & 0,12 & 0,37 & 1,93 & 5,18 & 13,85 & 27,09 & 6,69 \\
\hline Dedicado a las labores & & & & & & & & \\
\hline del hogar & 2,58 & 12,62 & 17,78 & 22,60 & 33,72 & 31,71 & 22,15 & 20,97 \\
\hline Estudiante & 54,02 & 6,33 & 1,16 & 0,48 & 0,25 & 0,15 & 0,04 & 6,53 \\
\hline Dedicado al voluntariado & 0,33 & 0,80 & 0,67 & 0,77 & 1,12 & 0,78 & 1,28 & 0,83 \\
\hline
\end{tabular}


TABLA 1 (cont.)

DESCRIPCIÓN DE LAS VARIABLES POR GRUPOS DE EDAD

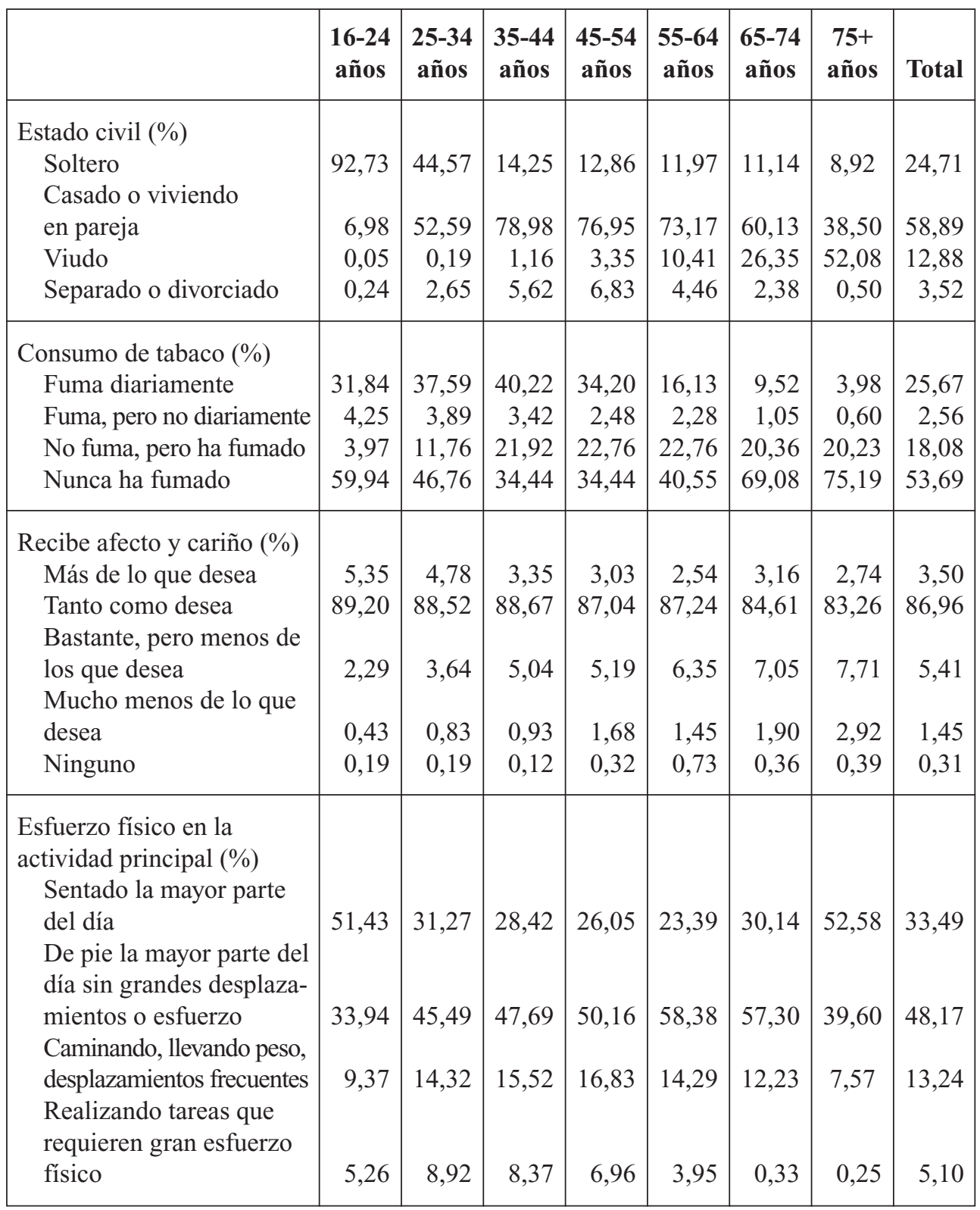


dades socioeconómicas en salud. Se han definido cuatro categorías que describen el estado civil del entrevistado: soltero ( $24,7 \%$ de la muestra), casado o que vive en pareja $(58,9 \%)$, viudo $(12,9 \%)$ y separado o divorciado $(3,5 \%)$. El sexo también aparece como variable explicativa puesto que las mujeres tienden a declarar peor salud que los hombres.

Para captar la influencia de los estilos de vida nocivos sobre las desigualdades en salud se ha incluido en el análisis información sobre el consumo de tabaco. En caso de que tenga impacto sobre las desigualdades, el resultado esperado es que ser fumador debería aumentar las desigualdades (habrá más variabilidad en el estado de salud), mientras que no haber fumado nunca o haber dejado de fumar deberían tener el efecto contrario. También intentamos incorporar la variable «índice de masa corporal» como resumen de los hábitos alimenticios y de ejercicio físico, pero la ausencia de información afectaba a muchos casos y nos hacía perder muchas observaciones. En cambio, hemos incluido una variable sobre el esfuerzo físico en la actividad principal, y otra que muestra la situación afectiva del entrevistado.

\section{Resultados}

La Tabla 2 muestra la distribución de los tres indicadores de salud utilizados por grupos de edad y quintiles de renta, así como los correspondientes índices de concentración $(I C)$. Dichos índices de concentración, junto con los intervalos de confianza para cada franja de edad se reflejan en la Figura 2. El IC del estado de salud autopercibido es positivo y significativamente diferente de cero en todos los casos, indicando que la buena valoración de la salud está relativamente más concentrada en los quintiles de renta más elevados (recuérdese lo dicho al comentar la Figura 1). La magnitud de la desigualdad es muy baja para el grupo de edad más joven, luego va aumentando con la edad hasta llegar a un máximo para el grupo entre $55 \mathrm{y}$ 64 años —el valor del $I C$ es 0,0710 — y a partir de ahí desciende, formando casi una $\mathrm{U}$ invertida, aunque con solapamientos en los intervalos de confianza, que indican que las diferencias entre estos grupos de edad no son significativas. Los amplios intervalos de confianza también indican que a estas edades hay mucha variabilidad en la percepción de la propia salud por niveles de renta, bastante más que para las edades más jóvenes.

La elevada desigualdad en el grupo previo a la edad oficial de jubilación (55-64 años) queda también patente observando en la Tabla 2 la diferencia en la proporción de individuos que declara tener buena o muy buena salud por quintiles de renta. Sólo un $45,5 \%$ de los individuos del primer quintil dice tener buena salud, mientras que dicho porcentaje alcanza el $69,1 \%$ en el último quintil. Es decir, hay una diferencia de casi 25 puntos entre el quintil más pobre y el más rico. La Figura 3 destaca estas diferencias entre los dos quintiles extremos de renta. Otra manera de expresar las diferencias en el estado de salud entre pobres y ricos es observando que hay la misma proporción de gente (un 69\%) que dice tener buena salud entre los ricos de 55-64 años 


\section{TABLA 2}

DISTRIBUCIÓN DE LA SALUD (TRES INDICADORES) POR GRUPOS DE EDAD Y QUINTILES DE RENTA Y SUS CORRESPONDIENTES ÍNDICES DE CONCENTRACIÓN

\begin{tabular}{|c|c|c|c|c|c|c|c|c|}
\hline \multicolumn{9}{|c|}{ Proporción de individuos con buena o muy buena salud } \\
\hline Quintiles de renta & $\begin{array}{c}16-24 \\
\text { años }\end{array}$ & $\begin{array}{c}25-34 \\
\text { años }\end{array}$ & $\begin{array}{c}35-44 \\
\text { años }\end{array}$ & $\begin{array}{c}45-54 \\
\text { años }\end{array}$ & $\begin{array}{l}55-64 \\
\text { años }\end{array}$ & $\begin{array}{c}65-74 \\
\text { años }\end{array}$ & $\begin{array}{c}75+ \\
\text { años }\end{array}$ & Total \\
\hline $20 \%$ más pobre & 0,8544 & 0,8148 & 0,6960 & 0,5845 & 0,4547 & 0,3789 & 0,3002 & 0,5603 \\
\hline $20-40 \%$ & 0,8589 & 0,8117 & 0,7803 & 0,7000 & 0,4801 & 0,3970 & 0,3357 & 0,6083 \\
\hline $40-60 \%$ & 0,8783 & 0,8133 & 0,8173 & 0,6876 & 0,5136 & 0,4449 & 0,3754 & 0,6283 \\
\hline $60-80 \%$ & 0,8541 & 0,8843 & 0,8277 & 0,7194 & 0,5598 & 0,4187 & 0,3623 & 0,6758 \\
\hline $20 \%$ más rico & 0,8828 & 0,8827 & 0,8414 & 0,7694 & 0,6915 & 0,5226 & 0,4342 & 0,7776 \\
\hline Total & 0,8657 & 0,8414 & 0,7926 & 0,6921 & 0,5399 & 0,4324 & 0,3615 & 0,6491 \\
\hline$\chi^{2}$ & 2,73 & $28,75^{*}$ & $72,18^{*}$ & $53,48^{*}$ & $77,45^{*}$ & $34,08^{*}$ & $24,13^{*}$ & $521,41^{*}$ \\
\hline $\mathrm{IC}^{* *}$ & 0,0143 & 0,0285 & 0,0242 & 0,0519 & 0,0710 & 0,0564 & 0,0619 & 0,0603 \\
\hline Desv.Estd. & 0,0014 & 0,0014 & 0,0015 & 0,0025 & 0,0039 & 0,0042 & 0,0054 & 0,0013 \\
\hline \multicolumn{9}{|c|}{ Proporción de individuos con al menos una enfermedad crónica } \\
\hline Quintiles de renta & $\begin{array}{l}16-24 \\
\text { años }\end{array}$ & $\begin{array}{c}25-34 \\
\text { años }\end{array}$ & $\begin{array}{c}35-44 \\
\text { años }\end{array}$ & $\begin{array}{l}45-54 \\
\text { años }\end{array}$ & $\begin{array}{l}55-64 \\
\text { años }\end{array}$ & $\begin{array}{l}65-74 \\
\text { años }\end{array}$ & $\begin{array}{c}75+ \\
\text { años }\end{array}$ & Total \\
\hline $20 \%$ más pobre & 0,2220 & 0,2886 & 0,3792 & 0,5523 & 0,7663 & 0,8436 & 0,8899 & 0,5898 \\
\hline $20-40 \%$ & 0,2584 & 0,2948 & 0,3480 & 0,4806 & 0,7572 & 0,8421 & 0,8757 & 0,5714 \\
\hline $40-60 \%$ & 0,2029 & 0,2994 & 0,3202 & 0,4831 & 0,6987 & 0,8341 & 0,8968 & 0,5619 \\
\hline $60-80 \%$ & 0,2632 & 0,2407 & 0,3376 & 0,4645 & 0,7011 & 0,8599 & 0,8579 & 0,5201 \\
\hline $20 \%$ más rico & 0,2273 & 0,2469 & 0,3009 & 0,4177 & 0,6116 & 0,7967 & 0,8790 & 0,4182 \\
\hline Total & 0,2347 & 0,2741 & 0,3372 & 0,4797 & 0,7070 & 0,8353 & 0,8798 & 0,5323 \\
\hline$\chi^{2}$ & 6,06 & $10,19^{*}$ & $13,48^{*}$ & $23,27^{*}$ & $40,58^{*}$ & $10,69^{*}$ & 4,72 & $328,20^{*}$ \\
\hline & $-0,0068$ & $-0,0423$ & $-0,0437$ & $-0,0507$ & $-0,0441$ & $-0,0085$ & $-0,0053$ & $-0,0621$ \\
\hline Desv.Estd. & 0,0230 & 0,0167 & 0,0123 & 0,0107 & 0,0069 & 0,0045 & 0,0039 & 0,0037 \\
\hline \multicolumn{9}{|c|}{ Proporción de individuos con limitaciones en la actividad habitual por enfermedades crónicas } \\
\hline Quintiles de renta & $\begin{array}{c}16-24 \\
\text { años }\end{array}$ & $\begin{array}{c}25-34 \\
\text { años }\end{array}$ & $\begin{array}{c}35-44 \\
\text { años }\end{array}$ & $\begin{array}{c}45-54 \\
\text { años }\end{array}$ & $\begin{array}{c}55-64 \\
\text { años }\end{array}$ & $\begin{array}{c}65-74 \\
\text { años }\end{array}$ & $\begin{array}{c}75+ \\
\text { años }\end{array}$ & Total \\
\hline $20 \%$ más pobre & 0,0549 & 0,0833 & 0,1353 & 0,2238 & 0,3062 & 0,3248 & 0,4547 & 0,2413 \\
\hline $20-40 \%$ & 0,0670 & 0,0741 & 0,0983 & 0,1548 & 0,2645 & 0,3519 & 0,3890 & 0,2044 \\
\hline $40-60 \%$ & 0,0501 & 0,0802 & 0,0844 & 0,1530 & 0,2886 & 0,3152 & 0,3665 & 0,2083 \\
\hline $60-80 \%$ & 0,0742 & 0,0648 & 0,0879 & 0,1548 & 0,2736 & 0,3298 & 0,3712 & 0,1848 \\
\hline $20 \%$ más rico & 0,0646 & 0,0540 & 0,0822 & 0,1129 & 0,1833 & 0,2877 & 0,3648 & 0,1240 \\
\hline Total & 0,0621 & 0,0713 & 0,0976 & 0,1599 & 0,2632 & 0,3219 & 0,3893 & 0,1926 \\
\hline$\chi^{2}$ & 2,66 & 561 & $18,91^{*}$ & $29,55^{*}$ & $25,53^{*}$ & 6,66 & $13,55^{*}$ & $209,60^{*}$ \\
\hline IC & 0,0236 & $-0,0826$ & $-0,1080$ & $-0,1177$ & $-0,0833$ & $-0,0340$ & $-0,0463$ & $-0,1140$ \\
\hline Desv.Estd. & 0,0500 & 0,0368 & 0,0274 & 0,0240 & 0,0179 & 0,0143 & 0,0137 & 0,0078 \\
\hline
\end{tabular}

Notas: * Estadísticamente significativos al 5\%.

** Este índice de concentración se refiere a la variable continua calculada a partir de las cinco categorías de autoevaluación de la salud, tal como se explicó en el apartado de descripción y tratamiento de los datos, y no a la variable ordinal original en la que están basados los porcentajes de individuos con buena o muy buena salud. 
FIGURA 2

ÍNDICES DE CONCENTRACIÓN DE

CADA UNO DE LOS TRES INDICADO-

RES DE SALUD POR GRUPOS DE EDAD E INTERVALOS DE CONFIANZA
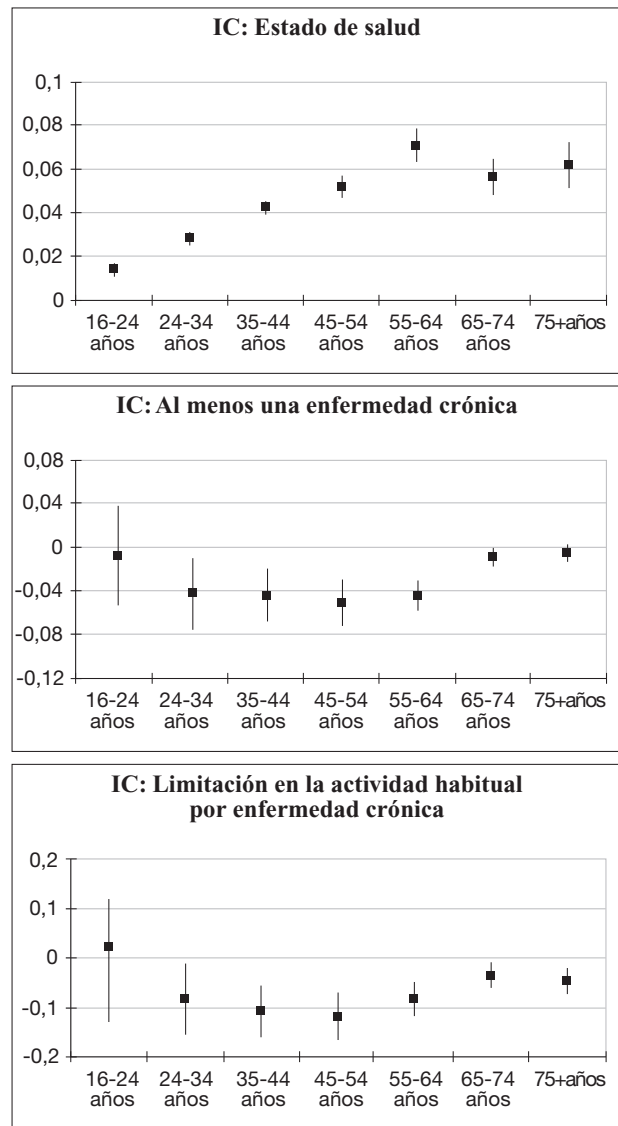

Fuente: Encuesta Nacional de Salud, 2003.
FIGURA 3

PERFILES DE SALUD

DE LOS QUINTILES

MÁS POBRE Y MÁS RICO

POR GRUPOS DE EDAD
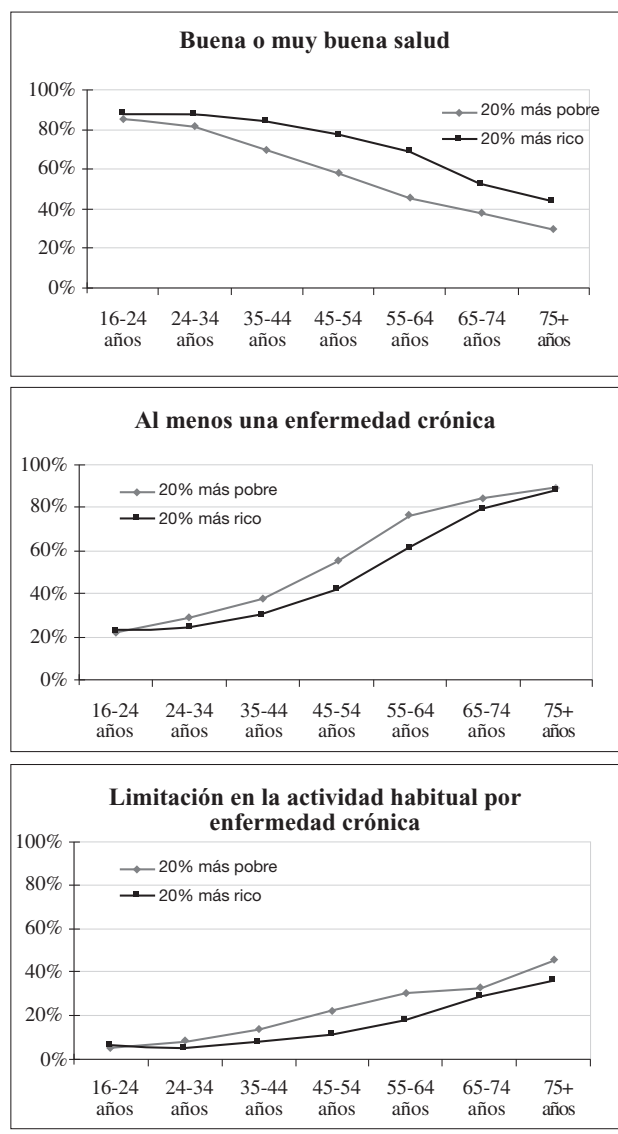

Fuente: Encuesta Nacional de Salud, 2003.

que entre los pobres veinte años más jóvenes (entre 35 y 44 años). Por cierto, cabe señalar que hasta los 64 años más de la mitad de la población (un 54\%) dice tener buena salud.

Cuando el indicador de salud es la presencia de al menos una enfermedad crónica hay tres grupos de edad (los dos extremos más la franja de 65-74 años) para los que los índices de concentración no son significativamente diferentes de cero. Por tanto, se entiende que para estos grupos no existe desigualdad asociada a renta. De hecho, vemos en la Tabla 2 que alrededor de un $88 \%$ de los mayores de 75 años declaran padecer al menos una enfermedad crónica, independientemente del quintil de renta al 
que pertenezcan. Para los otros cuatro grupos de edad los índices son muy similares entre sí, con valores entre de $-0,04$ y $-0,05$. El signo negativo indica que la presencia de enfermedades crónicas está relativamente más concentrada en los más pobres. La Figura 3 pone de relieve que la diferencia máxima entre los dos quintiles extremos de renta se da de nuevo para el grupo de edad previo a la jubilación. En los grupos de edad más mayores y más jóvenes no hay apenas diferencias, lo cual es consistente con que los índices de concentración no sean significativamente diferentes de cero para esos grupos. Finalmente, obsérvese que el mayor cambio en la prevalencia de enfermedades crónicas para la población en general se da a partir de los 55 años, cuando casi un $71 \%$ de la población dice padecer al menos uno de los trastornos nominados.

En cuanto a las limitaciones en las actividades habituales, la imagen que dibujan los valores del $I C$ es bastante similar a la del indicador anterior, apareciendo de nuevo un perfil en forma de $\mathrm{U}$ invertida si prescindimos del signo y tomamos los valores absolutos. Para el grupo de edad más joven el $I C$ no es significativamente diferente de cero; la desigualdad aumenta hasta llegar a un máximo para la franja entre 45 y 54 años y luego desciende para los dos grupos de edad más elevada, aunque con solapamientos en los intervalos de confianza. La variabilidad en este indicador es altísima para los dos grupos de edad más jóvenes. Por otro lado, la mayor concentración de las limitaciones en el primer quintil de renta para casi todos los grupos de edad es bastante evidente si se observa la Tabla 2.

Una vez conocidas las desigualdades, la siguiente cuestión que analizamos es: ¿cuál es la importancia de las diferentes características individuales para las desigualdades socioeconómicas en salud en cada grupo de edad? Recordemos (ecuación 6) que las variables explicativas pueden tener un efecto positivo o negativo sobre el grado de desigualad dependiendo del signo de su impacto sobre la salud (la elasticidad) y de su distribución según el nivel de renta (el índice de concentración). Las Tablas 3 y 4 muestran los resultados más relevantes del ejercicio de descomposición de los índices de concentración. En concreto, en la Tabla 3 se puede ver, para cada grupo de edad, el porcentaje del IC explicado por cada una de las variables o determinantes y en la Tabla 4 aparece la descomposición en sus dos partes (la elasticidad y el grado de concentración de la propia variable) para las variables con mayor grado de contribución según la Tabla 3. Destaca, en primer lugar, la renta, que explica entre el $41 \%$ y el $75 \%$ de la desigualdad hallada para los diferentes grupos de edad. La interpretación de estos porcentajes es como sigue: la desigualdad socioeconómica en el estado de salud percibido, por ejemplo, de los jóvenes entre 16 y 24 años, seria, ceteris paribus, un 75\% inferior si la renta estuviera distribuida igualitariamente entre todos los individuos de ese grupo de edad, o si la renta no tuviera ningún impacto diferencial sobre el estado de salud percibido. La mayor parte de la contribución se debe, en general, a la elasticidad y no tanto al índice de desigualdad en la propia renta (véase Tabla 4). La variabilidad en la contribución se debe sobre todo a las diferencias que hay en el impacto que tiene la renta sobre el estado de salud declarado, bastante más alto en el caso de los más mayores $\left(\eta_{\text {renta }}=1,1542\right)$. De hecho, el efecto de la renta sobre la salud es creciente con la edad, lo cual es un resultado interesante en sí mismo. 
TABLA 3

CONTRIBUCIÓN DE CADA VARIABLE A LA EXPLICACIÓN DE LA DESIGUALDAD SOCIOECONÓMICA EN SALUD AUTOPERCIBIDA, POR GRUPOS DE EDAD, 2003

\begin{tabular}{|c|c|c|c|c|c|c|c|}
\hline \multicolumn{8}{|c|}{ Como porcentaje del IC de la salud autopercibida } \\
\hline & $\begin{array}{c}16-24 \\
\text { años }\end{array}$ & $\begin{array}{c}25-34 \\
\text { años }\end{array}$ & $\begin{array}{c}35-44 \\
\text { años }\end{array}$ & $\begin{array}{c}45-54 \\
\text { años }\end{array}$ & $\begin{array}{c}55-64 \\
\text { años }\end{array}$ & $\begin{array}{c}65-74 \\
\text { años }\end{array}$ & $\begin{array}{c}75+ \\
\text { años }\end{array}$ \\
\hline Mujer & $-1 \%$ & $0 \%$ & $1 \%$ & $3 \%$ & $2 \%$ & $-2 \%$ & $-7 \%$ \\
\hline Enfermedad crónica & $1 \%$ & $5 \%$ & $6 \%$ & $11 \%$ & $14 \%$ & $6 \%$ & $4 \%$ \\
\hline Haber tenido accidente & $-2 \%$ & $1 \%$ & $1 \%$ & $0 \%$ & $0 \%$ & $0 \%$ & $-1 \%$ \\
\hline Logaritmo de la renta & $75 \%$ & $57 \%$ & $46 \%$ & $41 \%$ & $41 \%$ & $48 \%$ & $57 \%$ \\
\hline $\begin{array}{l}\text { Educación } \\
\text { Primaria } \\
\text { Secundaria } \\
\text { Universitaria }\end{array}$ & $\begin{array}{r}-14 \% \\
22 \% \\
14 \%\end{array}$ & $\begin{array}{r}-11 \% \\
-9 \% \\
52 \%\end{array}$ & $\begin{array}{r}-15 \% \\
-8 \% \\
59 \%\end{array}$ & $\begin{array}{r}-13 \% \\
5 \% \\
45 \%\end{array}$ & $\begin{array}{r}-6 \% \\
9 \% \\
30 \%\end{array}$ & $\begin{array}{r}2 \% \\
15 \% \\
24 \%\end{array}$ & $\begin{array}{r}8 \% \\
9 \% \\
19 \%\end{array}$ \\
\hline $\begin{array}{l}\text { Situación laboral } \\
\text { Parado } \\
\text { Jubilado } \\
\text { Percibe pensión por } \\
\text { invalidez } \\
\text { Percibe otras pensiones } \\
\text { Labores del hogar } \\
\text { Estudiante } \\
\text { Dedicado al voluntariado }\end{array}$ & $\begin{array}{r}3 \% \\
0 \% \\
0 \% \\
-2 \% \\
0 \%\end{array}$ & $\begin{array}{l}4 \% \\
0 \% \\
3 \% \\
1 \% \\
1 \%\end{array}$ & $\begin{array}{l}4 \% \\
0 \% \\
4 \% \\
0 \% \\
1 \%\end{array}$ & $\begin{array}{l}4 \% \\
0 \% \\
4 \% \\
0 \% \\
0 \%\end{array}$ & $\begin{array}{r}3 \% \\
0 \% \\
3 \% \\
-1 \% \\
6 \% \\
0 \% \\
1 \%\end{array}$ & $\begin{array}{r}0 \% \\
-1 \% \\
4 \% \\
-5 \% \\
5 \% \\
0 \% \\
0 \%\end{array}$ & $\begin{array}{r}0 \% \\
-2 \% \\
1 \% \\
3 \% \\
-1 \% \\
0 \% \\
0 \%\end{array}$ \\
\hline $\begin{array}{l}\text { Estado civil } \\
\text { Soltero } \\
\text { Viudo } \\
\text { Separado o divorciado }\end{array}$ & $\begin{array}{r}-3 \% \\
0 \% \\
0 \%\end{array}$ & $\begin{array}{r}-6 \% \\
0 \% \\
0 \%\end{array}$ & $\begin{array}{l}0 \% \\
0 \% \\
0 \%\end{array}$ & $\begin{array}{l}1 \% \\
0 \% \\
0 \%\end{array}$ & $\begin{array}{l}2 \% \\
0 \% \\
0 \%\end{array}$ & $\begin{array}{l}8 \% \\
0 \% \\
0 \%\end{array}$ & $\begin{array}{l}4 \% \\
3 \% \\
0 \%\end{array}$ \\
\hline $\begin{array}{l}\text { Consumo de tabaco } \\
\text { Fuma diariamente } \\
\text { Fuma, pero no diariamente } \\
\text { No fuma, pero ha fumado }\end{array}$ & $\begin{array}{l}0 \% \\
0 \% \\
0 \%\end{array}$ & $\begin{array}{r}0 \% \\
0 \% \\
-1 \%\end{array}$ & $\begin{array}{r}1 \% \\
0 \% \\
-1 \%\end{array}$ & $\begin{array}{l}0 \% \\
0 \% \\
0 \%\end{array}$ & $\begin{array}{l}0 \% \\
0 \% \\
0 \%\end{array}$ & $\begin{array}{l}0 \% \\
0 \% \\
0 \%\end{array}$ & $\begin{array}{l}0 \% \\
0 \% \\
1 \%\end{array}$ \\
\hline $\begin{array}{l}\text { Recibe afecto y cariño } \\
\text { Más de lo que desea } \\
\text { Tanto como desea } \\
\text { Bastante, pero menos de lo } \\
\text { que desea } \\
\text { Mucho menos de lo que } \\
\text { desea }\end{array}$ & $\begin{array}{r}0 \% \\
-1 \% \\
0 \% \\
0 \%\end{array}$ & $\begin{array}{l}0 \% \\
0 \% \\
0 \% \\
0 \%\end{array}$ & $\begin{array}{l}0 \% \\
0 \% \\
0 \%\end{array}$ & $\begin{array}{l}0 \% \\
0 \% \\
0 \% \\
0 \%\end{array}$ & $\begin{array}{c}0 \% \\
-1 \% \\
0 \% \\
0 \%\end{array}$ & $\begin{array}{r}0 \% \\
0 \% \\
-1 \% \\
-1 \%\end{array}$ & $\begin{array}{r}0 \% \\
2 \% \\
-3 \% \\
-3 \%\end{array}$ \\
\hline $\begin{array}{l}\text { Esfuerzo físico en la actividad ppal. } \\
\text { De pie la mayor parte del día } \\
\text { sin grandes esfuerzos } \\
\text { Caminando, llevando peso, } \\
\text { desplazamientos frecuentes } \\
\text { Gran esfuerzo físico }\end{array}$ & $\begin{array}{l}0 \% \\
0 \%\end{array}$ & $\begin{array}{r}-1 \% \\
0 \% \\
0 \%\end{array}$ & $\begin{array}{r}-1 \% \\
0 \% \\
-1 \%\end{array}$ & $\begin{array}{r}-2 \% \\
-1 \% \\
0 \%\end{array}$ & $\begin{array}{r}-3 \% \\
0 \% \\
0 \%\end{array}$ & $\begin{array}{r}-5 \% \\
3 \% \\
0 \%\end{array}$ & $\begin{array}{l}3 \% \\
3 \% \\
0 \%\end{array}$ \\
\hline Total & $100 \%$ & $100 \%$ & $100 \%$ & $100 \%$ & $100 \%$ & $100 \%$ & $100 \%$ \\
\hline
\end{tabular}


TABLA 4

DESCOMPOSICIÓN DE LA CONTRIBUCIÓN DE LAS PRINCIPALES VARIABLES QUE EXPLICAN LA DESIGUALDAD SOCIOECONÓMICA EN SALUD AUTOPERCIBIDA, POR GRUPOS DE EDAD, 2003

\begin{tabular}{|l|c|c|c|c|c|c|c|}
\hline $\begin{array}{l}\text { Índices de concentración, } \\
\boldsymbol{C}_{\boldsymbol{k}}\end{array}$ & $\begin{array}{c}\mathbf{1 6 - 2 4} \\
\text { años }\end{array}$ & $\begin{array}{c}\mathbf{2 5 - 3 4} \\
\text { años }\end{array}$ & $\begin{array}{c}\mathbf{3 5 - 4 4} \\
\text { años }\end{array}$ & $\begin{array}{c}\mathbf{4 5 - 5 4} \\
\text { años }\end{array}$ & $\begin{array}{c}\mathbf{5 5 - 6 4} \\
\text { años }\end{array}$ & $\begin{array}{c}\mathbf{6 5 - 7 4} \\
\text { años }\end{array}$ & $\begin{array}{c}\mathbf{7 5 +} \\
\text { años }\end{array}$ \\
\hline Enfermedad crónica & $-0,0067$ & $-0,0424$ & $-0,0437$ & $-0,0507$ & $-0,0441$ & $-0,0085$ & $-0,0053$ \\
\hline Logaritmo de la renta & 0,0337 & 0,0403 & 0,0364 & 0,0383 & 0,0375 & 0,0300 & 0,0303 \\
\hline $\begin{array}{l}\text { Educación } \\
\text { Primaria } \\
\text { Secundaria } \\
\text { Universitaria }\end{array}$ & $-0,1802$ & $-0,2474$ & $-0,3306$ & $-0,2330$ & $-0,0609$ & 0,0202 & 0,0800 \\
\hline & 0,0380 & $-0,0464$ & $-0,0584$ & 0,0520 & 0,1564 & 0,3361 & 0,4401 \\
Elasticidad, $\boldsymbol{h}_{\boldsymbol{k}}$ & 0,0842 & 0,2241 & 0,4460 & 0,5153 & 0,5931 & 0,6985 & 0,7614 \\
\hline Enfermedad crónica & $\mathbf{1 6 - 2 4}$ & $\mathbf{2 5 - 3 4}$ & $\mathbf{3 5 - 4 4}$ & $\mathbf{4 5 - 5 4}$ & $\mathbf{5 5 - 6 4}$ & $\mathbf{6 5 - 7 4}$ & $\mathbf{7 5 +}$ \\
$\mathbf{a n ̃ o s}$ & $\mathbf{a n ̃ o s}$ & $\mathbf{a n ̃ o s}$ & $\mathbf{a n ̃ o s}$ & $\mathbf{a n ̃ o s}$ & $\mathbf{a n ̃ o s}$ & $\mathbf{a n ̃ o s}$ \\
\hline Logaritmo de la renta & $-0,0256$ & $-0,0346$ & $-0,0544$ & $-0,1117$ & $-0,2337$ & $-0,3721$ & $-0,4928$ \\
\hline $\begin{array}{l}\text { Educación } \\
\text { Primaria } \\
\text { Secundaria } \\
\text { Universitaria }\end{array}$ & 0,3250 & 0,4058 & 0,5314 & 0,5591 & 0,7823 & 0,9060 & 1,1542 \\
\hline
\end{tabular}

La segunda contribución más substancial es la de la educación universitaria. En este caso, el factor más importante es la desigualdad (a favor de los ricos) en esta variable, con un índice de concentración que aumenta de manera notoria a medida que crece la edad. La educación secundaria tiene índices de concentración muy inferiores a los de la educación universitaria, pero llama la atención el signo positivo del índice de concentración en la franja de edad más joven, mientras que los dos grupos de edad que van de los 25 a los 44 años exhiben signo negativo. Es decir, para esos dos grupos de edad la educación secundaria (y la primaria) está relativamente más concentrada en los más pobres, mientras que en los más jóvenes (16-24 años) tener educación secundaria ¡«es de ricos»!, indicativo, dicho sea de paso, de que el conocido fracaso escolar en la educación secundaria obligatoria está concentrado en los pobres. Para los grupos de edad (25-34 y 35-44 años) que tienen índice de concentración negativo, la contribución de la educación secundaria a la desigualdad sale negativa: 9 y 8 por ciento, respectivamente según se puede ver en la Tabla 3. Es decir, en estos dos casos la educación secundaria contribuye a reducir la desigualdad. 
En tercer lugar de importancia encontramos la variable «enfermedad crónica». En todos los casos su contribución es positiva (aumenta la desigualdad) porque el índice de concentración es negativo (mayor prevalencia de trastornos crónicos entre los pobres) y el impacto sobre la salud también es negativo (y creciente con la edad). El porcentaje del $I C$ explicado por la presencia de enfermedades crónicas varía mucho, siendo menor para los grupos de edad extremos. Entre los más jóvenes, porque la prevalencia de condiciones crónicas es igualmente baja para todos los niveles de renta y la elasticidad también es baja ${ }^{4}$. En los grupos de edad más avanzada, aunque el impacto sobre la salud es alto $\left(\eta_{\text {enf. crónica }}=-0,4928\right.$ para los mayores de 75 años) el índice de concentración es muy bajo: las dolencias de carácter crónico afectan a la gran mayoría de los individuos independientemente de su renta.

El resto de variables tienen contribuciones menores - en muchos casos iguales a cero- $\mathrm{o}$ incluso negativas, como es la variable educación primaria o el hecho de pasar la mayor parte del día de pie, pero sin grandes esfuerzos. Una contribución negativa se debe interpretar como que la desigualdad hubiese sido incluso mayor sin la presencia de estas variables.

\section{Discusión}

La primera conclusión que se obtiene de nuestro análisis es que, si bien el deterioro de la salud (medida mediante cualquiera de los tres indicadores que hemos utilizado) es siempre mayor cuanto más avanzada es la edad, las desigualdades en salud no presentan esta senda progresiva. Las desigualdades en salud exhiben un perfil de U invertida, con un máximo entre los 45-54 ó 55-64 años. Este resultado es consistente con la mayor parte de la literatura revisada y mencionada en la introducción. La explicación sería que los factores biológicos, sociales y de comportamiento probablemente actúan de forma acumulativa con la edad, pero después de llegar a un cierto punto parece que hay otras cosas que cuentan y que podrían amortiguar las desigualdades.

Una de las razones para la disminución de las desigualdades en los mayores de 65 años es que a partir de la jubilación la dispersión en la renta (que no en el patrimonio) ya no es tan grande. En todo caso, la amplitud del intervalo de confianza del índice de concentración de la salud autopercibida para el grupo de edad más avanzada nos permitiría afirmar que a estas edades la renta no predice demasiado bien las diferencias en salud. Otra de las razones para el aplanamiento de las desigualdades parece estar en la situación laboral: estar parado, recibir una pensión de invalidez o dedicarse a las labores del hogar contribuyen a la ampliación de las desigualdades en las

4 Porqué la elasticidad de la salud con respecto a la presencia de al menos una enfermedad crónica es creciente con la edad es una incógnita para la que sería necesario buscar explicación mediante un análisis más detallado del que aquí se ha realizado. 
edades previas a la jubilación, mientras que su efecto desaparece o disminuye a partir de los $65 \mathrm{y}$, sobre todo, los 75 años. También podría ser que los principales factores asociados a la percepción de la salud en la vejez - que según Aspiazu Garrido et al. (2002) son el estado de salud mental y la capacidad funcional - estuvieran bastante igualitariamente distribuidos a esa edad entre los diferentes quintiles de renta. Una última razón podría ser que la inequidad en la utilización de servicios sanitarios fuera menor para estos grupos de edad, lo cual hemos comprobado mediante cálculos realizados para un trabajo aún no publicado.

En cuanto a que el principal factor que contribuye a la desigualdad es la propia renta, este resultado coincide con el hallado por Van Doorslaer y Koolman (2004) para los once países estudiados, entre ellos, España. En su trabajo, la educación superior también tiene una contribución muy elevada, aunque en general no tanto como en el nuestro. Al respecto, cabría especular acerca de cómo serán las desigualdades en un futuro, cuando las nuevas generaciones, en las que la educación superior está más generalizada, lleguen a esa edad previa a la jubilación. Si todo lo demás se mantuviera igual, las desigualdades en salud deberían disminuir, puesto que el índice de concentración de la educación superior en la franja de edad 25-34 es menos de la mitad del de esa franja entre 55 y 64 años $(0,2241$ frente a 0,5931$)$. Pero lo cierto es que nada se mantiene igual en el tiempo y esa especulación puede no resultar cierta.

Esta reflexión nos lleva a resaltar de nuevo que nuestro trabajo ofrece sólo una fotografía puntual de la situación de las desigualdades en salud por grupos de edad en 2003. Por consiguiente, no predetermina que los jóvenes de hoy experimenten los mismos procesos ni exhiban las mismas desigualdades cuando sean ancianos que las que observamos hoy para ese grupo de edad. Al no tratarse de un estudio longitudinal, nuestros datos no nos permiten separar los efectos del envejecimiento de los efectos cohorte ni de los efectos del período de análisis (Sacker et al., 2005). Así, no es lo mismo tener 75 años hoy (haber nacido en 1932) que tener 75 años en 2035 (haber nacido en 1960). La situación económica, los avances de la medicina, las experiencias vitales son muy diferentes para cada cohorte y las relaciones entre salud y edad cambian con el tiempo (Lawlor y Sterne, 2007), por lo que no sabemos cómo envejecerán (qué variables afectarán más a la salud) nuestros jóvenes de hoy.

Por la misma razón, es seguro que parte de las desigualdades observadas hoy son el resultado de experiencias pasadas (tanto más variadas y acumuladas cuanto más avanzada sea la edad), pero eso tampoco lo pueden detectar nuestros datos. Por ejemplo, los individuos que en 2003 pertenecen a un determinado quintil de renta pueden haber pertenecido a otros quintiles (más ricos o más pobres) en el pasado, pero desconocemos si ha sido así y qué poso ha dejado.

Lo que sí es cierto es que las relaciones cambian con el tiempo, y que el estudio de las desigualdades para toda una población puede esconder niveles de desigualdad muy diferentes para diferentes grupos de edad, por lo que un análisis pormenorizado, como el que se ha hecho aquí, puede ser útil en la detección y posible reducción de dichas desigualdades. 


\section{Referencias bibliográficas}

[1] ASPIAZU GARRIDO, M., CRUZ, A., VIllAGRASA, J.R., ABANADES, J.C., GARCÍA, N., ALVEAR, F. (2002): «Factores asociados a mal estado de salud percibido o a mala calidad de vida en personas mayores de 65 años», Revista Española de Salud Pública 76(6), pp. 683-699.

[2] BENACH, J., GARCÍA, M.D., CHAMIZO, H., BORREL, C., ROIG, A. (1996): «Desigualdades sociales en la mortalidad en España», en NAVARRO V., BENACH J., Las desigualdades sociales en salud (Informe de la Comisiión Científica de Estudio de las Desigualdades Sociales de Salud en España), Ministerio de Sanidad y Consumo, Madrid.

[3] BORREL, C., BENACH, J. (coordinadores) (2003): Les desigualtats en la salut a Catalunya. Mediterrània, Barcelona.

[4] BORREL, C., ROHLFS, I., ARTAZCOZ, L., MUNTANER, C. (2004): «Desigualdades en salud según la clase social en las mujeres. ¿Cómo influye el tipo de medida de la clase social?», Gaceta Sanitaria 18(supl.2), pp. 75-82.

[5] BURSTRÖM, B., FREDLUND, P. (2001): «Self-rated health: is it a good predictor of subsequent mortality among adults in lower as well as in higher social classes?», Journal of Epidemiology and Community Health 55(11), pp. 836-840.

[6] CANTARERO, D. PASCUAL, M. (2005): «Regional Differences in Health in Spain - An Empirical Analysis», ERSA conference papers ersa05p551, European Regional Science Association.

[7] CHANDOLA, T., FERRIE, J., SACKER, A., MARMOT, M. (2007): «Social inequalities in self reported health in early old age: follow-up of prospective cohort study», British Medical Journal 334, pp.990-996.

[8] DEATON, A., PASXON, C. (1998): «Ageing and inequality in income and health», AEA Papers and Proceedings, may, pp. 248-253.

[9] GARCÍA, P., LÓPEZ-NICOLÁS, A. (2007a): «Public and private health insurance and the utilisation of health care in Spain», Research on Economic Inequality 15, pp. 169-195.

[10] GARCÍA, P., LÓPEZ-NICOLÁS, A. (2007b): «Regional Differences in Socio-Economic Health Inequalities in Spain», Documento de trabajo no. 9/2007. Fundación BBVA.

[11] GARDNER, J., OSWALD, A. (2004): «How is mortality affected by money, marriage, and stress?», Journal of Health Economics 23, pp. 1181-1207.

[12] HU, Y., GOLDMAN, N. (1990): «Mortality differences by marital status: An international comparison», Demography 27, pp. 233-250.

[13] HUISMAN, M., KUNST, A., MACKENBACH, J. (2003): «Socioeconomic inequalities in morbidity among the elderly: A European overview», Social Science and Medicine 57, pp. 861-873.

[14] IDLER, E.L., BEYAMINI, Y. (1997): «Self-rated health and mortality: a review of twenty-seven community studies», Journal of Health and Social Behaviour 38, pp. 21-37.

[15] KAKWANI, N., WAGSTAFF, A., VAN DOORSLAER, E. (1997) «Socioeconomic inequality in health: measurement, computation and statistical inference», Journal of Econometrics 77, pp. 187-104 
[16] KUNST, A.E., GROENHOF, F., MACKENBACH, J., EU Working Group on Socioeconomic Inequalities in Health. (1998): «Occupational class and cause specific mortality in middle aged men in 11 European countries: comparison of population based studies», British Medical Journal 316, pp. 1636-1642.

[17] LAWLOR, D.A., STERNE, J.A.C. (2007): «Socioeconomic inequalities in health», British Medical Journal 334, pp. 963-964.

[18] MACKENBACH, J., KUNST, A.E. (1997): «Measuring the magnitude of socio-economic inequalities in health: An overview of available measures illustrated with two examples from Europe», Social Science and Medicine 44(6), pp. 757-771.

[19] MARMOT, M., DAVEY SMITH, G., STANSFELD, S., PATEL, C., NORTH, F., HEAD, J. et al. (1991): «Health Inequalities among British civil servants: the Whitehall II Study», Lancet 337, pp.1387-1393.

[20] MARMOT, M., SHIPLEY, M. (1996): «Do socio-economic differences in mortality persist after retirement? 25 years follow up of civil servants from the First Whitehall Study», British Medical Journal 313, pp. 1177-1180.

[21] MERLO, J., GERDTHAM, U.-G., LYNCH, J., BECKMAN, A., NORLUND, A., LITHMAN, T. (2003): «Social inequalities in health- do they diminish with age? Revisiting the question in Sweden 1999», International Journal of Equity in Health 2(2).

[22] NAVARRO, V., BENACH, J. (1996): Las desigualdades sociales en salud en España (Informe de la Comisión Científica de Estudio de las Desigualdades Sociales de Salud en España). Ministerio de Sanidad y Consumo, Madrid.

[23] REGIDOR, E., GUTIÉRREZ-FISAC, J.L., RODRÍGUEZ, C.(1994): Diferencias y desigualdades en salud en España. Díaz de Santos, Madrid.

[24] SACKER, A., CLARKE, P., WIGGINS, R.D., BARTLEY, M. (2005): «Social dynamics of health inequalities: A growth curve analysis of aging and self-assessed health in the British household panel survey 1991-2001», Journal of Epidemiology and Community Health 59, pp. 495-501.

[25] TOWNSEND, P., DAVIDSON, N., WHITEHEAD, M. (1992): Inequalities in Health. The Black Report and The Health Divide Penguin Books, 2nd edition, London.

[26] URBANOS, R. (2000): «Desigualdades sociales en salud y efectividad potencial de las políticas públicas: Un estudio aplicado con datos españoles», Hacienda Pública Española 154, pp. 217-238.

[27] VAN DOORSLAER, E., WAGSTFF, A., BLEICHRODT, H., CALONGE, S., GERDTHAM, U.-G., GERFIN et al. (1997): «Income-related inequalities in health: some international comparisons», Journal of Health Economics 16(1), pp. 93-112.

[28] VAN DOORSLAER, E., GERDTHAM, U.-G. (2003): «Does inequality in self-assessed health predict inequality in survival by income», Social Science and Medicine 57, pp. 1621-1629.

[29] VAN DOORSLAER, E., JONES, A. (2003): «Inequalities in self-reported health: validation of a new approach to measurement», Journal of Health Economics 22, pp. 61-87.

[30] VAN DOORSLAER, E., KOOLMAN, X. (2004): «Explaining the differences in income-related inequalities across European countries», Health Economics 13(7), pp. 609-628. 
[31] VAN DOORSLAER, E., KOOLMAN, X., JONES; A. (2004): «Explaining incomerelated inequalities in doctors utilisation in Europe», Health Economics 13(7), pp. 629-647.

[32] VAN OURTI, T. (2003): «Socio-economic inequality in ill-health amongst the elderly. Should one use current or permanent income?», Journal of Health Economics 22, pp. 219-241.

[33] VICTOR, C. (1989): «Inequalities in Health in Later Life», Age and Ageing vol. 18(2), pp. 387-391.

[34] WAGSTFF, A., PACI, P., VAN DOORSLAER, E. (1991): «On the measurement of inequality in health», Social Science and Medicine 33(5), pp.545-557.

[35] WAGSTAFF, A., VAN DOORSLAER, E., WATANABE, N. (2003): «On decomposing health sector inequalities, with an application to malnutrition inequalities in Vietnam», Journal of Econometrics 112, pp. 1207-223.

[36] WAGSTAFF, A., VAN DOORSLAER, E. (2000): Equity in health care finance and delivery, en CULYER, A.J., NEWHOUSE, J.P. (eds). Elsevier, Amsterdam.

[37] WEST, P. (1997): «Health inequalities in the early years: is there equalisation in youth?», Social Science and Medicine 44, pp. 833-858.

[38] WILKINSON, R. (1996): Unhealthy Societies: the Afflictions of Inequality. Routledge, London.

[39] WILKINSON, R. (1997): «Health inequalities: Relative or absolute material standards», British Medical Journal 314, pp. 591-595.

[40] WYKE, S., FORD, G. (1992): «Competing explanations for associations between marital status and health», Social Science and Medicine 34(5), pp. 523-532. 